\title{
Fibroma osificante juvenil, presentación de un caso clínico y revisión de la literatura
}

\author{
Juvenil Ossifying Fibroma, case report and review of the literature
}

José T. San Martín $\mathbf{M}^{3}$, José Tomás Andrade $\mathrm{D}^{2}$, María de los Ángeles Baeza $\mathrm{A}^{2}$, César Toro $\mathrm{A}^{1}$.

\section{RESUMEN}

Se presenta el caso de una paciente de seis años, de sexo femenino sin antecedentes mórbidos ni familiares de importancia, quien consulta por proptosis izquierda en abril de 2011. Se realiza resonancia magnética de cerebro evidenciándose un tumor de seno etmoidal con compromiso de órbita izquierda que ocasiona exoftalmo. La biopsia endoscópica nasal fue compatible con fibroma osificante juvenil.

Posteriormente se realiza antrostomía con etmoidectomía en dos tiempos, con resultados satisfactorios para la paciente.

Aunque el fibroma osificante juvenil es un tipo de lesión benigna poco frecuente, puede llegar a ser muy agresiva y con una alta tasa de recidiva en ciertas ocasiones, por lo que se debe tener en cuenta para realizar un diagnóstico y tratamiento precoz, con un seguimiento programado a largo plazo.

Palabras clave: Seno etmoidal, fibroma osificante, fibroma osificante juvenil, fibroma cemento osificante, tumor benigno fibro-óseo.

\begin{abstract}
This paper presents the case of a patient of six years old female without background or important morbidly in her family, who consults for left-sided proptosis in April of 2011. It performs brain magnetic resonancebeing demonstrated a tumor of theethmoid sinus with commitment to the left orbit that causes exophthalmus. Nasal endoscopic biopsy was compatible with juvenile ossifying fibroma. It was subsequently performed ananthrostomy whitethmoidectomy in two times, with satisfactory results for the patient.

Although the Juvenile ossifying fibroma is a rare type of benign lesion, can be very aggressive and have a high recurrence rate in certain occasions, by what should be taken into account to make an early diagnosis and treatment, with a scheduled follow up in the long term.

Key words: Ethmoid sinus, ossifying fibroma, juvenile ossifying fibroma, cement ossifying fibroma, benign fibro-osseous tumor.
\end{abstract}

Médico. Departamento Otorrinolaringología, Hospital Sótero del Río.

2 Médico. Departamento Otorrinolaringología, Pontificia Universidad Católica de Chile.

3 Médico Cirujano. 


\section{INTRODUCCIÓN}

La lesión benigna intraósea denominada fibroma osificante corresponde a una etiología poco frecuente ${ }^{1-6}$, que en casos aislados afecta los senos etmoidales ${ }^{6}$.

Fue descrita por primera vez por Benjamins en 1938. Años más tarde Lichenstein describió los tumores de características osteofibrosas ubicados en cabeza y cuello ${ }^{5}$.

Está constituida por tejido fibroso celular, calcificaciones esféricas y estructuras óseas orientadas al azar y no se han reportado diferencias significativas según sexo ${ }^{3}$.

Se ha reportado una tasa de recurrencia entre $30 \%-58 \% 4,6,13,14$, sin datos sobre transformación maligna $^{4}$.

El fibroma osificante es una patología benigna que a pesar de su condición puede crecer 0 comprometer al paciente de manera agresiva por su crecimiento local 6 .

\section{CASO CLÍNICO}

Se presenta el caso de una paciente de sexo femenino de 6 años de edad, procedente de Santiago de Chile, sin antecedentes mórbidos ni familiares de importancia, quien fue referida al CRS de Otorrinolaringología del Hospital Sótero del Río en abril de 2011 desde una clínica externa, por proptosis izquierda progresiva de meses de evolución. Presentaba movimiento ocular conservado, sin diplopia ni otras alteraciones en la visión.

La paciente traía una resonancia magnética de cerebro con énfasis en órbitas y complemento con tomografía computarizada, la cual evidenció una lesión expansiva bien delimitada centrada en celdillas etmoidales a izquierda de $23 \times 30 \times 22$ $\mathrm{mm}$, con áreas hiperintensas en T2 de impregnación irregular con contraste. La lesión expandía la lámina papirácea, introduciéndose en el aspecto posterior de la órbita, desplazando el músculo recto medial determinando exoftalmo (Figuras $1 \mathrm{y}$ 2). El diagnóstico diferencial inicialmente incluyó hemangioma sinonasal y fibroma osificante. Se realizó biopsia endoscopía nasal donde se observó un tumor de consistencia blanda y aspecto polipoideo en relación al techo nasal y lado del cornete medio de la fosa nasal izquierda.

El reporte histopatológico fue compatible con tumor de células gigantes sin signos de malignidad.

Por la condición de la paciente, dado el énfasis en manejo multidisciplinario, fue evaluada por oftalmología, quien luego de examen físico y revisión de imágenes no evidenció compromiso estético ni funcional del ojo izquierdo, motivo por el cual no se indicó cirugía oftalmológica.

Dado los hallazgos clínicos, radiológicos e histopatológicos la paciente fue intervenida quirúrgicamente de manera endoscópica en julio de 2011. Durante el procedimiento se evidenció la presencia de un tumor de consistencia blanda, muy friable y sangrante, que se ubicaba en zona etmoidal y deformaba completamente el meato medio, llegando hasta la pared medial nasal. Se resecó tumor en forma progresiva, quedando remanente de lesión en pared lateral nasal, posterior a apertura de ostium maxilar y en techo etmoidal,

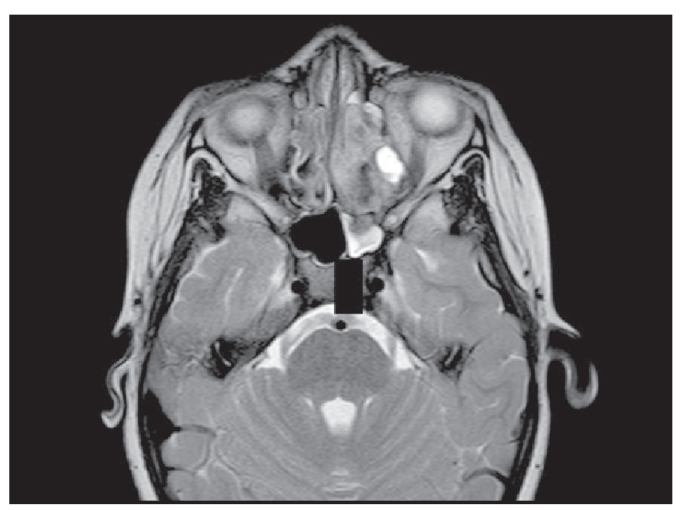

Figura 1. RNM cerebro.

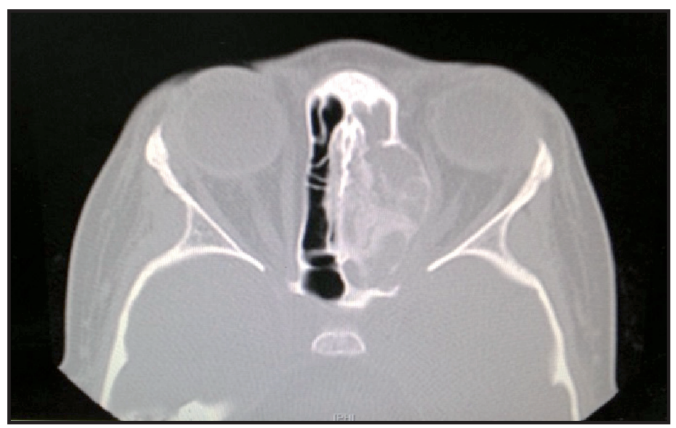

Figura 2. TAC de cerebro con énfasis en órbitas. 
Io cual se postergó para un segundo tiempo operatorio.

Se envió el tejido resecado a análisis histopatológico, donde se observaron células gigantes multinucleadas de tipo osteoblasto en trabéculas, hallazgos compatibles con fibroma osificante juvenil. Se examina biopsia anterior en conjunto y el aspecto histopatológico fue coincidente.

En marzo de 2012, se realizó segundo tiempo operatorio, donde se observó lesión poliposa frontoetmoidal que se extrajo, se resecó la mucosa y el remanente de tejido óseo fibroso de la pared lateral y medial. Luego de lo cual la paciente no presentó complicaciones y se dio de alta con control seriado en policlínico de ORL. Se decidió además tomar imágenes de control con TAC de cerebro, los cuales demostraban una clara resolución del cuadro (Figuras 3 y 4).

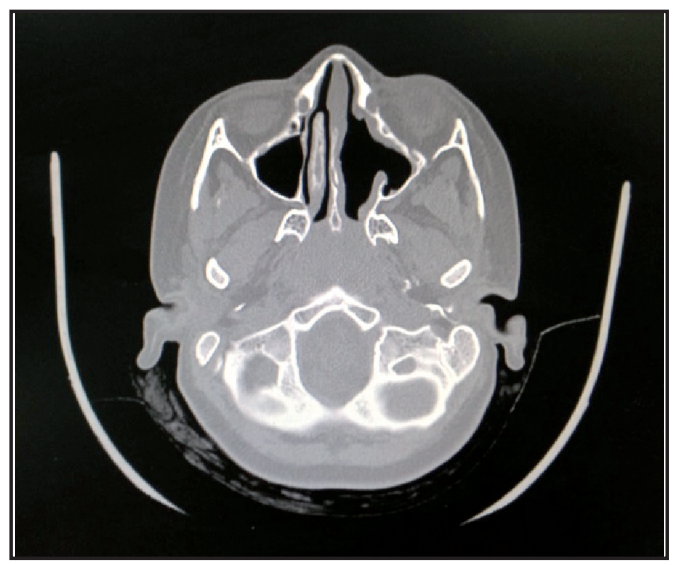

Figura 3. TAC cerebro.

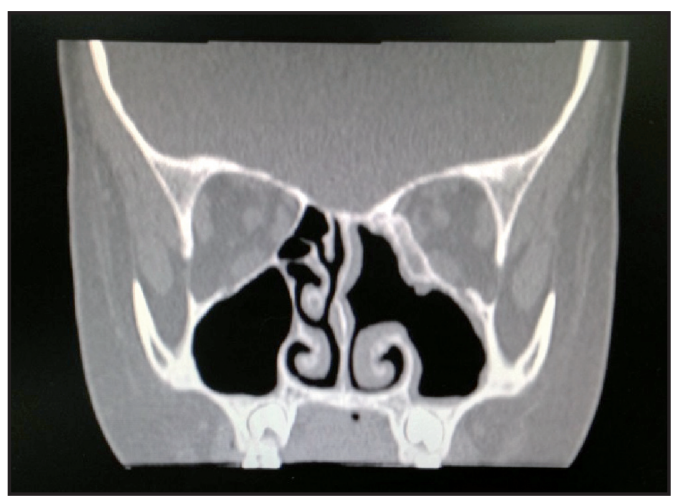

Figura 4. TAC cerebro.

\section{REVISIÓN DE LA LITERATURA}

La lesión benigna intraósea denominada fibroma osificante corresponde a una etiología poco frecuente ${ }^{1-6}$, que en casos aislados afecta los senos etmoidales ${ }^{6}$. Está constituida por tejido fibroso celular, calcificaciones esféricas y estructuras óseas orientadas al azar.

En 1971 las lesiones derivadas del cementum fueron clasificadas en cuatro entidades ${ }^{7}$.

- Displasia cemental periapical

- Cementoma gigantiforme

- Cementoma benigno

- Fibroma cementificante

El fibroma osificante es parte del último.

En la literatura existen diversos términos para referirse a éste, tales como fibroma osificante activo juvenil, fibroma osificante samomatoide, desmoosteoblastoma, fibroma osificante samomatoide agresivo, etc, lo cual aún es motivo de controversia.

El fibroma osificante no tiene una etiología precisada. Dentro de las hipótesis planteadas en la literatura está la presencia de un hamartoma del tejido óseo, restos embrionarios localizados 0 el antecedente de traumatismos en la zona como factor predisponente ${ }^{8-9}$

Su presentación clínica es variable dependiendo de la zona donde esté ubicado. En la gran mayoría de los casos reportados se ubica en la mandíbula, generando deformidad de ésta, siendo la presentación en seno etmoidal extremadamente $\operatorname{rara}^{3,6}$.

Si se compromete el globo ocular puede generar exoftalmo, diplopia, y alteración en la agudeza

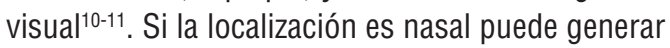
obstrucción nasal uni o bilateral, anosmia, hiposmia, epistaxis o rinorrea. En localización etmoidal puede haber dolor retroocular o nasal. En caso de bloquear los complejos osteomeatales puede generar rinosinusitis y sus complicaciones ${ }^{5-6,10-11}$. Si hay invasión intracraneal puede provocar fístula de líquido cefalorraquídeo, meningitis, absceso cerebral dentro de otros.

Además de la clínica, el apoyo con imágenes tales como tomografía computarizada y resonancia magnética es fundamental para el apoyo tanto del diagnóstico como de sus diferenciales. Dando 
una idea de la ubicación e invasión local para un adecuado enfrentamiento quirúrgico. Sin embargo, las características radiológicas de estos tumores varían notablemente en la literatura. En general, el aspecto radiológico depende de la madurez de la lesión, pero la mayoría los presenta como lesiones de densidad bien definida, mezclada con algunas zonas radiolúcidas. Además, presentan bordes radiográficos bien definidos, acompañados de esclerosis marginal y cortex delgado $0^{15,16}$.

Finalmente la histología es definitoria, mostrando trabéculas óseas rodeadas por osteoblastos $y$ en ocasiones osteoclastos ${ }^{2,6}$.

El tratamiento multidisciplinario facilita la resección de estos tumores ${ }^{12}$, como se mostró en nuestro caso. La resección quirúrgica nos permite un diagnóstico histopatológico. Idealmente ésta debe ser completa dada la alta tasa de recurrencia reportada en la literatura, que va desde $6 \%$ al $58 \%$ 4-6,13-14.

Basados en la mejoría del instrumental de endoscopía, como de la técnica de esta misma y la aplicación rutinaria de los precisos sistemas de navegación, los límites del abordaje endoscópico han disminuido considerablemente durante las últimas décadas ${ }^{17}$. Es con esto, que la resección endoscópica de los fibromas osificantes que afectan la región naso-sinusal, se convierte en una excelente opción terapéutica a la hora de elegir la vía de abordaje en estos pacientes, cuando es realizada por un cirujano experimentado. Algunas de las ventajas de este abordaje incluyen la visualización directa del tumor, la ampliación de la imagen, la no realización de incisiones externas, con la consecuente deformidad externa y la disminución de la morbilidad general. Pero es importante destacar algunas complicaciones como la lesión de base de cráneo, la cual puede resultar en una fuga de líquido cerebroespinal, que de todas maneras puede ser reparada dentro del mismo escenario quirúrgi$\mathrm{Co}^{18}$. El abordaje craneofacial se ha convertido en la vía quirúrgica más aceptada para la resección de tumores de mayor tamaño que han traspasado la fosa craneal anterior y que se extienden a la cavidad craneal ${ }^{19}$.

El manejo posoperatorio en estos casos debe incluir el control endoscópico, además del control imagenológico correspondiente, particularmente en los casos en los que la resección no ha podido ser completa, destacando como bien se dijo en un principio, que se trata de un tumor benigno, por lo que se debe de tratar de ser conservador en el tratamiento quirúrgico de éste, teniendo siempre en mente, que el seguimiento a largo plazo es fundamental, para así evitar posibles recidivas.

\section{CONCLUSIÓN}

En el presente trabajo se presenta un caso clínico característico de fibroma osificante juvenil en un paciente de 6 años de edad, además de la revisión de la literatura correspondiente. Todo, para alertar e instruir al clínico en la sospecha y tratamiento de esta entidad, además de tratar de demostrar el enfoque de un tratamiento quirúrgico adecuado, pero a la vez no agresivo cuando se realiza un seguimiento a largo plazo, que claramente es lo más recomendado en estos pacientes.

\section{BIBLIOGRAFÍA}

1. Benjamins CE. Das Osteoid-Fibroma mitaty pischer Verkalkungim Sinus frontalis. Acta Otolaryngol 1938; 26: 26-44.

2. Pathologic Quiz Case A 10 -Year-Old Boy With Swelling of the Left Maxilla Lt Col David L. Wells, USAF; MAJ Keith Kaplan, MC, USA. Arch Pathol Lab Med- Vol 127, August 2003.

3. Slootweg PJ, Panders AK, Koopmans R, Nikkels $P G$. Juvenile ossifying fibroma.An analysis of 33 cases with emphasis on histopathological aspects. J Oral Pathol Med 1994; 23: 385-8.

4. NeVILLE BW. Oral \& Maxillofacial Pathology. $2^{\text {nd }} \mathrm{ed}$. Philadelphia, Pa: WB Saunders; 2002: xv, 843.

5. Lichtenstein I, Jafee. Fibrous dysplasia of bone. Arch Pathology 1942; 33: 777-816.

6. José Eduardo Guzmán Durán, Santiago Hernández González. Ethmoidal Ossifying Fibroma, A Pediatric Case. Acta de Orl y Cirugía de Cabeza y Cuello 2008, Vol $36 \mathrm{~N}^{\circ} 1$.

7. Pindborg J, Kramer IRH. Histological typing of odontogenic tumors, jaw cysts and allied lesions, in: International Histological Classification of Tumors, vol. 5, World Health Organization, 1971; 31-4.

8. Santaolalla F, Sánchez Ana et al. ORL-DIPS 2002; 29 (3): 124-7. 
9. Martín-Granizo R, Sánchez-Cuellar A, Falahat F. Cemento ossifying fibroma of the upper gingivae. Otolaryngol Head Neck Surg 2000; 122: 775.

10. Shields JA, Peyster RG, Handler SD, Augsburger $\mathrm{JJ}$, KaPUSTIAK J. Massive juvenile ossifying fibroma of maxillary sinus with orbital involvement. $\mathrm{Br} \mathrm{J}$ Ophthalmol 1985; 69: 392e5.

11. Hachach-Haram, Sarah Benyon, Sarah Maling, Naresh Joshi, William E. Grant, W. Niall A. KIRKPATRICK. Surgical management of two complex cases of large juvenile orbital ossifying fibroma Nadine. Journal of Plastic, Reconstructive \& Aesthetic Surgery 2011; 64: 1661e1664.

12. Hartstein MR, Grove AS, Woog JJ, Shore JW, JOSEPH MP. The multidisciplinary management of psammomatoid ossifying fibroma of the orbit. Ophthalmology 1998; 105(4): 591e5.

13. Zachariades N, Vairaktaris E, Papanicolau S, Triantafyllou D, Papavassiliou D, Mezitis M. Ossifying fibroma of the jaws. Review of the literature and report of 16 cases. Int J Oral Surg 1984; 13: 1-6.

14. Su L, Weathers DR, Waldron CA. Distinguishing features of focal cementoosseous dysplasia and cemento-ossifying fibromas II. A clinical and radiologic spectrum of 316 cases. Oral Surg Oral Med Oral Pathol Oral Radiol Endod 1997; 84: $540-9$.

15. DS MacDonald-Jankowski. Cemento-ossifying fibromas inthe jaws of Hong Kong Chinese. Dentomaxillofacial Radiology 1988; 27(5): 298304.

16. CA WaLdRon. Fibro-osseous lesions of the jaws. Journal of Oral and Maxillofacial Surgery 1993; 51(8): 828-35.

17. Ledderose GJ, Stelter K, Becker S, Leunig A. Paranasal ossifying fibroma: endoscopic resection or wait and scan? Eur Arch Otorhinolaryngol 2011; 268(7): 999-1004.

18. Post G, Kountakis SE. Endoscopic resection of large sinonasal ossifying fibroma. Am J Otolaryngol 2005; 26(1): 54-6.

19. Noudel R, Chauvet E, Cahn V, Merol JC, Chays A, Rousseaux P. Transcranial resection of a large sinonasal juvenile psammomatoid ossifying fibroma. Childs Nerv Syst 2009; 25(9): 1115-20. 